\section{Reining in renin}

\section{By Tim Fulmer, Senior Writer}

Although antihypertensives have become the standard of care in diabetic nephropathy, the mechanistic link between hyperglycemia and blood pressure, as well as between hyperglycemia and potential downstream renal damage, has remained elusive. ${ }^{1}$

A paper published in The Journal of Clinical Investigation has now identified a $\mathrm{G}$ protein-coupled receptor (GPCR) in the kidneys that may link diabetic hyperglycemia to hypertension. Antagonizing the receptor could thus be a new strategy to treat or prevent nephropathy in diabetes patients.

The JCI paper builds on previous research that identified the metabolic intermediate succinate as a ligand for GPR91, a GPCR that is highly expressed in the kidneys. ${ }^{2}$ The new work, by researchers at the University of Southern California (USC), suggests that GPR91 mediates renin release by the kidney under high-glucose conditions. ${ }^{3}$

In healthy people, renin activates the reninangiotensin system (RAS) to help maintain stable blood pressure and fluid homeostasis. In renal disease, high levels of renin lead to chronic activation of RAS. This, in turn, causes hypertension, edema, proteinuria and, over time, diabetic nephropathy that can lead to end-stage renal disease (see Figure 1, "Linking diabetic hyperglycemia to kidney disease").

The authors of the JCI paper therefore tested whether disrupting GPR91 expression could lower renin levels.

In kidney preparations isolated from healthy GPR91 knockout mice, renin levels were significantly lower following glucose perfusion than those in kidney preparations from wild-type mice $(p<0.001)$. Similarly, in GPR91 knockout mice with type 1 diabetes, total renin content was significantly lower in the kidney than it was in the kidneys of wild-type mice $(p<0.05)$.

Urine samples and whole-kidney homogenates isolated from the diabetic mice showed significantly higher levels of succinate than samples from nondiabetic control mice $(p<0.001)$.

Thus, the authors concluded, high glucose levels led to increased accumulation of succinate in the kidney, which activated GPR91. That activation caused the release of renin and the upregulation of RAS. The authors concluded that GPR91 "is a new potential therapeutic target to prevent renal complications of diabetes."

"A key insight of the JCI paper is that it suggests at least one mecha- nism by which diabetes could trigger a pathway that contributes to nephropathy," said Thomas Coffman, professor of medicine, cell biology and immunology and chief of nephrology at Duke University Medical Center and Durham VA Medical Center.

\section{Targeting GPR91}

Janos Peti-Peterdi, corresponding author on the JCI paper and associate professor of physiology, biophysics and medicine at USC, told SciBX that next steps include both therapeutic and diagnostic applications.

On the therapeutics front, "we are performing in vitro microperfusion assays on kidney preparations to identify small molecule compounds that inhibit GPR91 signaling and decrease or prevent renin release from the juxtaglomerular cells," he said. "We expect it will be safe to administer these compounds systemically or even orally, since GPR91 is expressed mainly in the kidney."

In the kidney, the juxtaglomerular cells synthesize and secrete renin.

On the diagnostics side, Peti-Peterdi said, "we have filed for a patent to use succinate as a biomarker for kidney damage, and we are now developing an assay that will accurately measure micromolar succinate levels in urine."

The goal of the assay will be to "determine a threshold concentration of succinate at which diabetes patients are at increased risk of diabetic nephropathy and kidney damage," he said. "Angiotensin-converting enzyme (ACE) inhibitors, angiotensin receptor blockers (ARBs) and potentially GPR91 inhibitors could then be introduced at the early onset of disease in order to prevent irreversible kidney damage and progression to end-stage renal disease."

However, according to Coffman, "not all diabetics get nephropathy, and furthermore, patients with diabetic nephropathy often do not have elevated renin levels. This suggests that other mechanisms are also at play at the interface between metabolic dysfunction and renal damage."

He added: "One way of getting a better understanding of what's going on might be to knock out GPR91 in other animal models of diabetes and metabolic disease and look at important indicators of renal dysfunction, such as tissue pathology and levels of albuminuria."

Albuminuria, measurable levels of the protein albumin in the urine, often occurs in nephropathy.

\section{Getting down to business}

Although recognizing the importance of the JCI findings, company researchers interviewed by SciBX also noted that further studies are needed to determine the relevance and universality of the GPR91 link for nephropathy in humans.

Klaus Dembowsky, managing director of Speedel's Speedel Experimenta preclinical research unit, was unsure whether succinate levels will be as predictive of metabolic disease in humans as they are in mice. 


\section{TARGETS \& MECHANISMS}

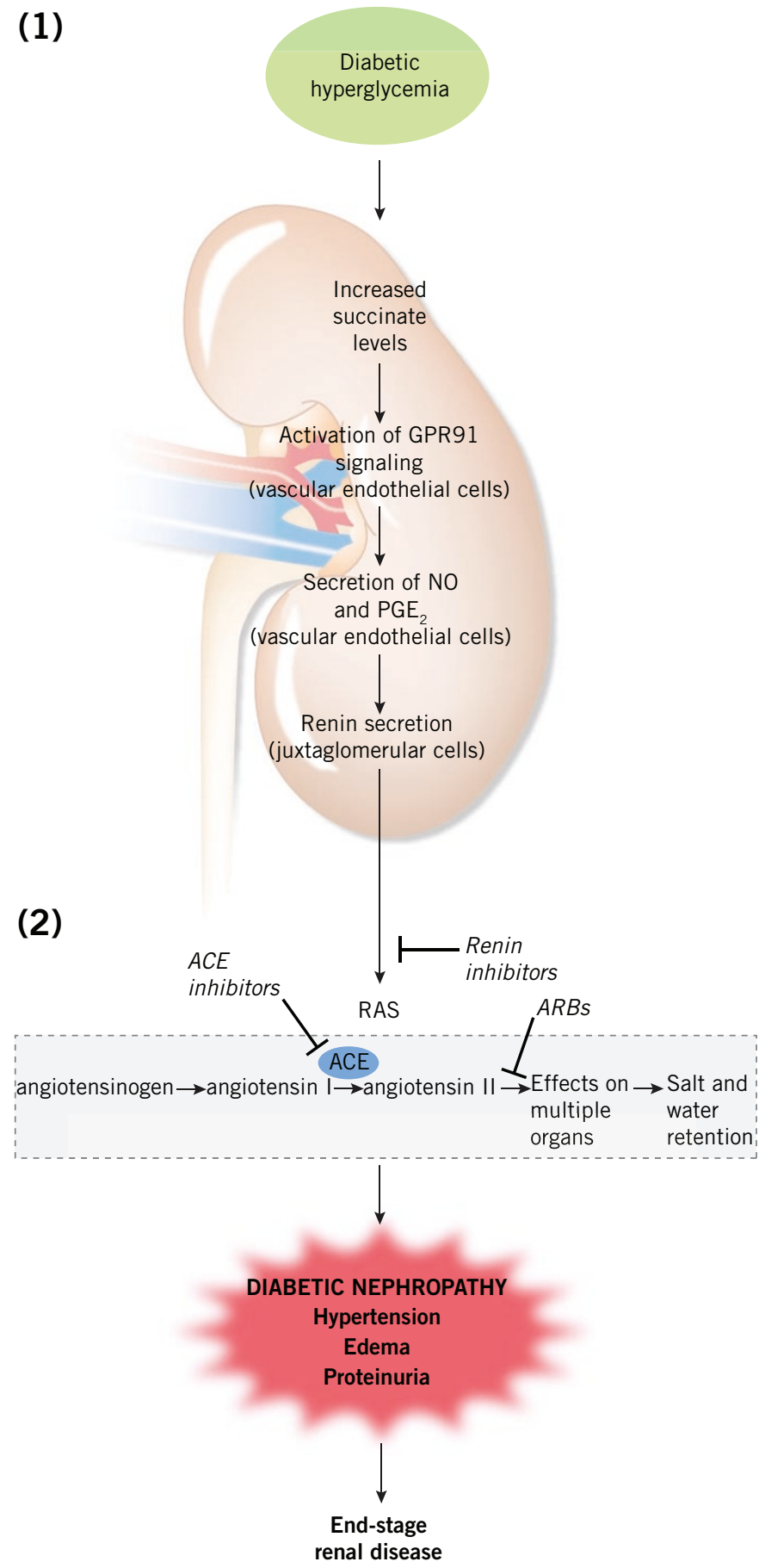

"Work published last year by a Pfizer Inc. group showed similarly increased plasma levels of succinate in rodent models of diabetes and hypertension," he said. " "However, these researchers also found no difference in plasma succinate levels between diabetes patients and healthy controls."

Given the dichotomy between rodent and human data, Dembowsky thinks the next steps for the USC researchers "should probably focus on finding proof of principle in humans or primates. They could, for example, compare plasma and urine succinate levels between healthy
Figure 1. Linking diabetic hyperglycemia to kidney disease. Research published in The Journal of Clinical Investigation ${ }^{3}$ suggests that G protein-coupled receptor 91 (GPR91), which is upstream of the renin-angiotensin system (RAS), could be a key mediator of hyperglycemia-induced renal hypertension in diabetes - and thus could be a potential target for preventing onset or slowing progression of diabetic nephropathy.

(1) In kidney preparations isolated from mice, increased glucose levels triggered release of renin by the following mechanism: high levels of glucose resulted in increased accumulation of the metabolic intermediate succinate in kidney tissue, which activated GPR91 signaling in vascular endothelial cells. Secretion of nitric oxide $(\mathrm{NO})$ and prostaglandin $\mathrm{E}_{2}\left(\mathrm{PGE}_{2}\right)$ by these same cells then triggered release of renin by adjacent juxtaglomerular cells.

(2) In diabetic nephropathy, aberrantly high levels of renin lead to chronic RAS activation, with resulting long-term hypertension, edema and high levels of serum protein in the urine (proteinuria). This condition can lead to end-stage renal disease (ESRD). Standard of care for diabetic nephropathy has thus aimed at reducing blood pressure through the use of antihypertensives to inhibit RAS activation. These include renin inhibitors such as the marketed Tekturna aliskiren from Speedel (SWX:SPPN) and Novartis AG (NYSE:NVS; SWX:NOVN) as well as angiotensin-converting enzyme (ACE) inhibitors and angiotensin receptor blockers (ARBs). A number of generic ACE inhibitors are available, and marketed ARBs include Atacand candesartan from AstraZeneca plc (LSE: AZN; NYSE:AZN), Avapro irbesartan from Bristol-Myers Squibb Co. (NYSE:BMY) and sanofi-aventis Group (Euronext:SAN; NYSE:SNY), Benicar olmesartan from Daiichi-Sankyo Co. Ltd. (Tokyo:4568; Osaka:4568), Cozaar losartan from Merck \& Co. Inc. (NYSE:MRK), Diovan valsartan from Novartis, and Micardis telmisartan from Boehringer Ingelheim GmbH.

volunteers and patients with diabetes or renal disease. Perhaps this would help show whether GPR91 signaling is increased in disease in humans and also whether succinate levels in the urine reflect that increase."

The key would be seeing if urine levels do predict nephropathy even if plasma levels do not.

Peti-Peterdi said his lab is indeed investigating succinate levels in the plasma and urine of humans.

Sophia Roy, senior director of Merck \& Co. Inc.'s Merck Research Laboratories unit and site lead in the cardiovascular diseases department, also thinks additional work in animals might be warranted. Next steps toward establishing GPR91 as a target in diabetic nephropathy could include "the evaluation of whether a similar paracrine signaling mechanism is operative in the whole animal, and more specifically, in an insulin-resistant diabetic animal where endothelial dysfunction is prominent," she said.

Paracrine signaling is a form of intercellular signaling in which the target cell is in close proximity to the signal-releasing cell. In the JCI paper, renin release from the juxtaglomerular cells was dependent on prior activation of GPR91 signaling by succinate in the adjacent vascular endothelial cells. 


\section{TARGETS \& MECHANISMS}

Peti-Peterdi noted that the endothelium-dependent paracrine signaling mechanism "is only one example of probably many GPR91-induced intrarenal signaling mechanisms that are important under pathological conditions including diabetes mellitus."

Future research will include identifying other regions of GPR91 signaling within the kidney, he said.

Tekturna aliskiren from Speedel and Novartis AG is the first approved renin inhibitor. The drug is sold to treat hypertension and has also been studied in the Phase III Aliskiren in the Evaluation of Proteinuria in Diabetes (AVOID) trial in patients with diabetic nephropathy. Data showed that aliskiren, in combination with the ARB losartan, lowered proteinuria levels by $20 \%$ compared with the effect of losartan alone.

"Speedel is also developing second-generation renin inhibitors with improved bioavailability and better tissue penetration. Three of these are in the clinic now for hypertension indications," Dembowsky said.
Cozaar losartan, an angiotensin II antagonist from Merck, is marketed to treat hypertension.

REFERENCES

1. Gurley, S. \& Coffman, T. Semin. Nephrol. 27, 144-152 (2007)

2. He, W. et al. Nature 429, 188-193 (2004)

3. Toma, I. et al. J. Clin. Invest.; published online June 5, 2008; doi:10.1172/JCl33293

Contact: Janos Peti-Peterdi, University of Southern California, Los Angeles, Calif.

e-mail: petipete@usc.edu

4. Sadagopan, N. et al. Am. J. Hypertens. 20, 1209-1215 (2007)

COMPANIES AND INSTITUTIONS MENTIONED

Duke University Medical Center, Durham, N.C.

Durham VA Medical Center, Durham, N.C.

Merck \& Co. Inc. (NYSE:MRK), Whitehouse Station, N.J.

Novartis AG (NYSE:NVS; SWX:NOVN), Basel, Switzerland

Pfizer Inc. (NYSE:PFE), New York, N.Y.

Speedel (SWX:SPPN), Basel, Switzerland

University of Southern California, Los Angeles, Calif. 